\title{
A coincidence between a hydrocarbon plasma absorption spectrum and the 15450 DIB
}

\author{
H. Linnartz ${ }^{1}$, N. Wehres ${ }^{1,2}$, H. Van Winckel $^{3}$, G. A. H. Walker ${ }^{4}$, D. A. Bohlender ${ }^{5}$, \\ A. G. G. M. Tielens ${ }^{6}$, T. Motylewski ${ }^{7}$, and J. P. Maier ${ }^{7}$ \\ 1 Raymond and Beverly Sackler Laboratory for Astrophysics, Leiden Observatory, Leiden University, PO Box 9513, \\ 2300 RA Leiden, The Netherlands \\ e-mail: wehres@strw.leidenuniv.nl \\ 2 Kapteyn Astronomical Institute, University of Groningen, PO Box 800, 9700 AV Groningen, The Netherlands \\ 3 Instituut voor Sterrenkunde, K.U. Leuven, Celestijnenlaan 200B, 3000 Leuven, Belgium \\ ${ }^{4}$ Physics and Astronomy Department, University of British Columbia, Vancouver, BC V6T 1Z4, Canada \\ 5 National Research Council of Canada, Herzberg Institute of Astrophysics, 5071 W. Saanich Road, Victoria, BC V9E 2E7, Canada \\ ${ }^{6}$ Leiden Observatory, Leiden University, PO Box 9513, 2300 RA Leiden, The Netherlands \\ 7 Department of Chemistry, University of Basel, Klingelbergstrasse 80, 4056 Basel, Switzerland
}

Received 8 January 2010 / Accepted 4 February 2010

\section{ABSTRACT}

\begin{abstract}
Aims. The aim of this work is to link the broad $\lambda 5450$ diffuse interstellar band (DIB) to a laboratory spectrum recorded through expanding acetylene plasma.

Methods. Cavity ring-down direct absorption spectra and astronomical observations of HD 183143 with the HERMES spectrograph on the Mercator Telescope on La Palma and the McKellar spectrograph on the DAO $1.2 \mathrm{~m}$ Telescope are compared.

Results. In the 543-547 nm region a broad band is measured with a band maximum at $545 \mathrm{~nm}$ and $F W H M$ of $1.03(0.1) \mathrm{nm}$ coinciding with a well-known diffuse interstellar band at $\lambda 5450$ with an $F W H M$ of $0.953 \mathrm{~nm}$.

Conclusions. A coincidence is found between the laboratory and the two independent observational studies obtained at higher spectral resolution. This result is important, as a match between a laboratory spectrum and a - potentially lifetime broadened - DIB is found. A series of additional experiments were performed in order to unambiguously identify the laboratory carrier of this band, but this was not successful. The laboratory results, however, restrict the carrier to a molecular transient, consisting of carbon and hydrogen.
\end{abstract}

Key words. astrochemistry - techniques: spectroscopic - ISM: molecules - ISM: lines and bands

\section{Introduction}

Diffuse interstellar bands are absorption features observed in starlight that is crossing diffuse interstellar clouds. Since this discovery in the beginning of the 20th century, scientists have been puzzled by the origin of these bands that appear both as relatively narrow and rather broad bands covering the UV/VIS and NIR (Tielens \& Snow 1995). In the last decennia, the idea has been established that it is unlikely that all these bands originate from one or a very few carriers, and with the progress of optical laboratory techniques, several families of potential carriers have been investigated. It was shown that the electronic transitions of a series of PAH-cations do not match the listed DIBs (Salama et al. 1996, 1999; Bréchignac \& Pino 1999; Ruiterkamp et al. 2002). Similarly, systematic laboratory studies of electronic spectra of carbon chain radicals have not resulted in positive identifications either (Motylewski et al. 2000; Ball et al. 2000a; Jochnowitz \& Maier 2008), even though it is known from combined radioastronomical and Fourier transform microwave (FTMW) studies that many of such species are present in dense clouds (Thaddeus \& McCarthy 2001). Only $\mathrm{C}_{3}$ has been recorded unambiguously in diffuse interstellar clouds (Maier et al. 2001).

Other studies, focusing on multi-photon excitation in molecular hydrogen (Sorokin et al. 1998), or spectra of fullerenes and nano-tubes (Kroto \& Jura 1992; Foing \& Ehrenfreund 1994) have been unsuccessful as well. In the past years, several coincidences between laboratory and astronomical DIB studies have been reported in the literature. These have all turned out to be accidental, and from a statistical point of view, the chance of an overlap is also quite substantial, because DIBs cover a major part of the wavelength region between roughly 350 and $1000 \mathrm{~nm}$. However, there are several conditions that have to be fulfilled before any coincidence of a laboratory and an astronomical DIB spectrum may be interpreted as a real match. These conditions have become stricter with the recent improvement in achievable spectral resolution, both in laboratory and astronomical studies.

The two most important DIB matching criteria to link laboratory and astronomical data follow:

1. The gas-phase laboratory and observational values of both peak position and bandwidth of the origin band transition should be identical, unless it can be argued that a spectral shift or band profile change may come from an isotope or temperature effect. An example of the latter is given by spectroscopic measurements on benzene plasma yielding an absorption feature coinciding with the strongest DIB at $442.9 \mathrm{~nm}$ (Ball et al. 2000b; Araki et al. 2004). The laboratory $F W H M$ turned out to be narrower than in the astronomical spectrum. It was argued that the spectrum of a non-polar molecule cooled in a molecular expansion may 
be considerably colder than in space, where only radiative cooling applies. A similar discussion has been given by (Motylewski et al. 2000), who show that unresolved rotational profiles may change substantially for different temperatures, as has also been calculated and discussed by CossartMagos \& Leach (1990).

2. Once the origin band overlaps with a DIB feature, gas-phase transitions to vibrationally excited levels in the electronically excited state of the same carrier molecule should match as well, and the resulting band profiles should behave in a similar way (i.e. with comparable equivalent width ratios) (Motylewski et al. 2000). A good example for this is the electronic spectrum of $\mathrm{C}_{7}^{-}$that has been regarded for several years as a potential carrier, because subsequent electronic bands fulfilled both conditions (Tulej et al. 1998; Kirkwood et al. 1998). Detailed follow-up studies show that the series of (near) matches was coincidental (McCall et al. 2001).

Despite much progress both from the observational and laboratory side, all efforts to assign DIBs have in the end resulted in a rather static situation, thereby triggering more and more exotic explanations for DIB carriers, and the origin of the DIBs is still as mysterious as it was nearly 100 years ago.

In this letter we report a match of a laboratory spectrum with a diffuse interstellar band that is special, because the first condition is fulfilled for a rather broad and potentially lifetime broadened DIB; i.e., the laboratory and astronomical spectra should be fully identical, independent of temperature restrictions. New astronomical observations obtained with the Mercator telescope, using the HERMES spectrograph and the Dominion Astrophysical Observatory (DOA) $1.2 \mathrm{~m}$ telescope, using the McKellar spectrograph, are presented in order to characterize the band profile of the $\lambda 5450$ DIB with the best possible resolution. Even though we have not been able to unambiguously identify the laboratory carrier, which is most likely a smaller hydrocarbon bearing molecular transient, we think that this overlap is important to report, since it provides a new piece in the puzzle.

\section{Laboratory experiments}

The experimental set-up is described in Linnartz et al. (1998) and Motylewski \& Linnartz (1999), and has been extensively used to study many carbon chain radicals of astrophysical interest (Jochnowitz \& Maier 2008). The monochromatic output $\sim 0.1 \mathrm{~cm}^{-1}$ at $540 \mathrm{~nm}\left(\sim 18500 \mathrm{~cm}^{-1}\right)$ of a pulsed dye laserbased cavity ring-down set-up is focused into an optical cavity consisting of two highly reflective mirrors $(R>0.9999)$. A special, pulsed high-pressure slit-nozzle system capable of producing intense $300 \mu$ s long plasma pulses by discharging $(-1 \mathrm{kV}$, $100 \mathrm{~mA})$ an expanding gas mixture of $1 \%$ acetylene $\left(\mathrm{C}_{2} \mathrm{H}_{2}\right)$ in $\mathrm{He}$ is mounted inside the cavity with its slit parallel to the optical axis of the cavity. In the expansion, a wide variety of new species is formed, and as the technique is not mass selective, special care has to be taken when assigning bands to specific carriers.

Mass selective matrix isolation spectra offer a good starting point for an assignment (Jochnowitz \& Maier 2008). In the case of rotationally resolved spectra, unambiguous identifications are generally possible, either by combination differences of accurate spectral fits, or by isotopic studies using $\mathrm{C}_{2} \mathrm{D}_{2}$ instead of $\mathrm{C}_{2} \mathrm{H}_{2}$ (or a mixture of $\mathrm{C}_{2} \mathrm{H}_{2} / \mathrm{C}_{2} \mathrm{D}_{2}$ ). The source runs at $30 \mathrm{~Hz}$, and special care is taken that the pressure inside the cavity remains constant during jet operation to reduce baseline fluctuations. Rotational temperatures are typically $T_{\text {rot }} \sim 10-20 \mathrm{~K}$. This low temperature results in a spectral simplification and



Fig. 1. The $\lambda 5450$ DIB. The top spectrum is a simulated spectrum available from DIB catalogues. The middle and bottom figures show observational spectra from the HERMES and McKellar spectrograph, respectively. The HERMES spectra show the $\lambda 5450$ DIB recorded toward HD 183143 and toward a reference star (HD 164353). The McKellar spectra show a reference spectrum toward Rigel (top), the DIB spectrum also toward HD 183143 (bottom), and the corresponding spectrum (middle) in which the SII stellar line has been deblended.

simultaneously increases the detection sensitivity because of improved state density. In addition, the source offers a Doppler free environment with a relatively long effective absorption path length. The laser beam intersects the $3 \mathrm{~cm}$ long planar expansion about 5-10 $\mathrm{mm}$ downstream using a sophisticated trigger scheme. Subsequent ring-down events (typically 20-30 $\mu$ s for a $52 \mathrm{~cm}$ long cavity) are recorded as a function of the laser frequency by a photo-diode and transferred to an averaged ringdown time by fitting 45 subsequent ring-down events. This value as function of the laser wavelength provides a sensitive way to record optical spectra. An absolute frequency calibration is obtained by recording an $I_{2}$ reference spectrum simultaneously.

\section{Astronomical observations}

The laboratory data are compared to observations from two different astronomical facilities.

\subsection{HERMES @ Mercator telescope}

The HERMES observations were carried out in service mode using the Mercator telescope at Roque de los Muchachos Observatory on La Palma. The $1.2 \mathrm{~m}$ telescope is operated by the Katholieke Universiteit in Leuven, Belgium, in collaboration with the Observatory in Geneva, Switzerland. The spectra were obtained in June 2009 with HERMES (High Efficiency and Resolution Mercator Echelle Spectrograph) (Raskin \& Van Winckel 2008), which is a fibre-fed-cross-dispersed spectrograph. The spectrograph has a fixed spectral format and samples the spectrum between 377 and $990 \mathrm{~nm}$ in 55 spectral orders on a $4.6 \mathrm{k} \times 2 \mathrm{k} \mathrm{CCD}$. The spectral resolution is slightly variable over the field, but is 85000 on average. We obtained 3 spectra of $1200 \mathrm{~s}$ of HD 183143 (B7Ia, $m(v)=6.92, B-V=+1.001$ ), the DIB spectral standard with a reddening $E(B-V)$ close to 1.0 . The reference star HD $164353(B 5 I b, m(v)=3.97, B-V=-0.002)$ was sampled in 3 exposures of $1 \mathrm{~min}$. The spectral reduction was performed using the specifically coded HERMES pipeline, and 
it contains all the standard steps in spectral reduction. The wavelength calibration is based on spectra of ThAr and Ne lamps. As we are mostly interested in the broad absorption feature that is centred around $545 \mathrm{~nm}$, we focus further on this spectral region of HD 183143. The spectra are shown in Fig. 1 (middle rows) and compared to the $\lambda 5450$ DIB profile as available from a series of digital DIB catalogues (Herbig 1975; Jenniskens \& Desert 1994; Tuairisg et al. 2000; Galazutdinov et al. 2000) in the upper row.

\subsection{McKellar@DAO telescope}

Fifty-five half-hour spectra were taken with the McKellar Spectrograph and SITe-4 CCD at the DAO $1.2 \mathrm{~m}$ telescope, operated by the National Research Council of Canada, over 6 nights between 16 and 23 July 2006 (UT) at a dispersion of $10.1 \AA / \mathrm{mm}$ giving $0.151 \AA$ /pixel for a resolution $\sim 0.3 \AA$. The data were processed in a standard fashion using IRAF $^{1}$. The aggregate spectrum had a signal-to-noise ratio of about 1200/pixel before correction of telluric lines. Removal of the quite weak telluric features was performed conventionally with spectra $(S / N \sim 1600)$ of the A0 V star zeta Aql (HD 177724) as the template.

Rigel, an unreddened comparison star with a B8 Ia spectral type very similar to the B7 Ia of HD 183143 was also observed to identify photospheric lines that contaminate the interstellar features observed in the latter star. The sharp line at approximately $5454 \AA$ arises from S II and was removed from the spectrum of HD 183143 by simply fitting a Voigt profile to the line and subtracting this from the original spectrum. The final "deblended" spectrum is plotted as a comparison in Fig. 1 (lower panel, middle spectrum).

\section{Results}

In Fig. 2 several spectra in the 543-547 nm region are compared. The top spectrum is the digital DIB spectrum of the $\lambda 5450$ DIB (Herbig 1975; Jenniskens \& Desert 1994; Tuairisg et al. 2000; Galazutdinov et al. 2000). The spectrum in the middle is a zoomin on the deblended McKellar spectrum as shown in Fig. 1. The bottom spectrum is the laboratory spectrum recorded in direct absorption through an expanding $1 \% \mathrm{C}_{2} \mathrm{H}_{2} / \mathrm{He}$ plasma. The similarity between the three spectra is striking.

This wavelength region was initially scanned to search for the ${ }^{1} \Pi_{u}-\mathrm{X}^{1} \Sigma_{g}{ }^{+}$electronic origin band spectrum of the linear carbon chain radical $\mathrm{C}_{7}$ (following the $\mathrm{C}_{7}^{-}$DIB discussion) that was located in matrix isolation experiments around $542.3 \mathrm{~nm}$. The laboratory spectrum, shown in Fig. 2, consists of many narrow lines that come from small acetylene fragments (typically $\mathrm{C}_{2}$ and $\mathrm{CH}$ ) that get weaker when the distance from the nozzle orifice to the optical axis is increased, but there is clearly a broad underlying feature. As this band shifts by $1.5 \mathrm{~nm}$ to the red upon $\mathrm{C}_{2} \mathrm{D}_{2}$ precursor substitution, it was initially neglected, because both $\mathrm{C}_{2} \mathrm{H}_{2}$ and $\mathrm{C}_{2} \mathrm{D}_{2}$ should result in an identical spectrum for $\mathrm{C}_{7}$. The shift is illustrated in Fig. 3. In addition, the deuterated spectrum appears to be somewhat stronger. Despite this negative result for $\mathrm{C}_{7}$, the profile hiding under the narrow lines in the $\mathrm{C}_{2} \mathrm{H}_{2}$ precursor experiment perfectly matches the $\lambda 5450 \mathrm{DIB}$

\footnotetext{
${ }^{1}$ IRAF is distributed by the National Optical Astronomy Observatory, which is operated by the Association of Universities for Research in Astronomy (AURA) under cooperative agreement with the National Science Foundation.
}

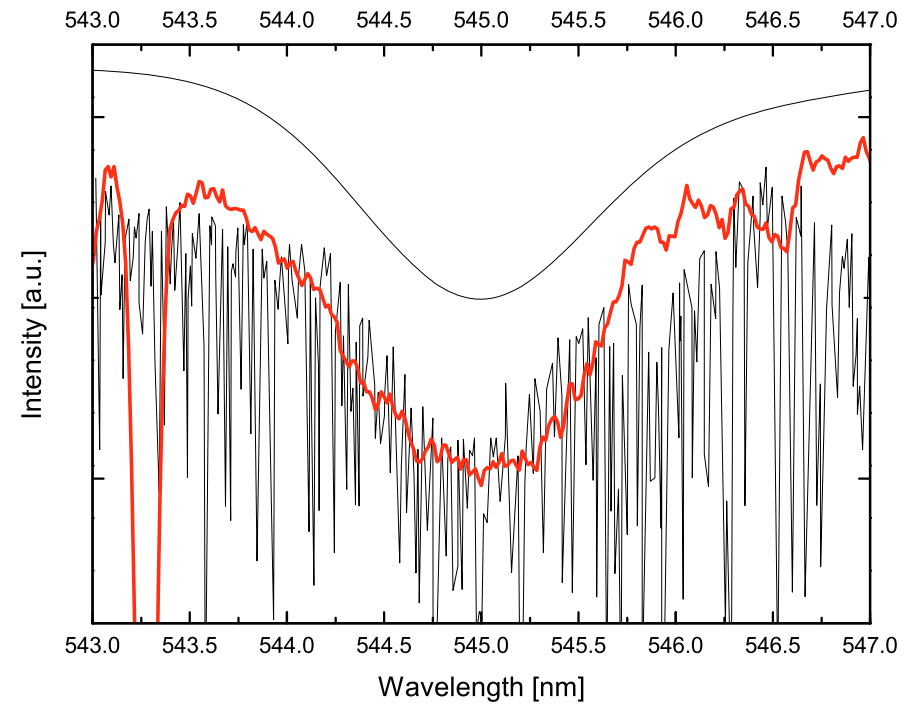

Fig. 2. The top spectrum shows the digital $\lambda 5450 \mathrm{DIB}$, the middle spectrum shows the deblended McKellar data and the bottom spectrum shows the laboratory cavity ring-down absorption spectrum through a supersonically expanding acetylene plasma.

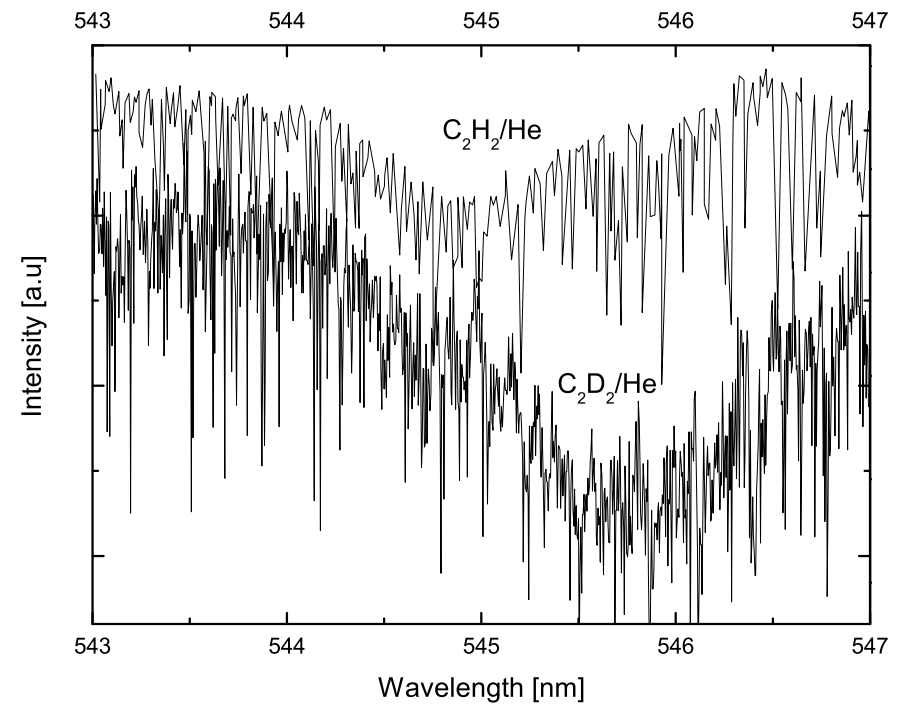

Fig. 3. Comparison between laboratory experiments sampling expanding plasma using regular acetylene (top) and deuterated acetylene (bottom) as a precursor gas.

available from the DIB databases, which is one reason additional observations were performed.

\section{Discussion}

There is little discussion possible about the coincidence between the recorded laboratory spectrum and the $\lambda 5450$ DIB. Both bands have a central peak position of $545 \mathrm{~nm}$ and an FWHM of $1.03(0.1) \mathrm{nm}$ (laboratory spectrum) and $0.953 \mathrm{~nm}$ (observational spectrum) (Tuairisg et al. 2000). The uncertainty in the first value comes from the overlap of the many individual transitions, which prohibits a clear view of the broad feature. The question is more whether this actually represents a DIB match, and for this complementary information is needed. Additional laboratory work was performed, where it should be noted that the scans shown in Figs. 2 and 3 typically last $45 \mathrm{~min}$ to an hour, in order to achieve the required sensitivity and to cover 
a frequency domain broad enough to differenciate band profile and base line; i.e., fast optimizations are impossible. The laboratory band does not show any structure that can be related to unresolved $\mathrm{P}, \mathrm{Q}$, and R-branches. With $1.03(0.1) \mathrm{nm}$, the band is also much broader than the unresolved rotational profile of a larger carbon chain radical. For comparison, at $15 \mathrm{~K}$, the band profile of the linear $\mathrm{C}_{6} \mathrm{H}$ radical (at $525 \mathrm{~nm}$ ) is about five times narrower (Linnartz et al. 1999). It should also be noted that such a broad feature actually represents a large absorption compared to many of the sharper DIBs. Changing the experimental settings to vary the final temperature in the expansion by measuring close $\left(\sim 50 \mathrm{~K}^{\prime} \mathrm{warm}^{\prime}\right)$ and far $\left(\sim 10 \mathrm{~K}^{\prime}\right.$ cold $\left.{ }^{\prime}\right)$ downstream does not substantially change the $F W H M$ of the spectral contour. As the narrow overlapping transitions have $F W H M$ s close to the laser bandwidth, experimental broadening artifacts, such as residual Doppler broadening in the expansion or amplified spontaneous emission, can be excluded. It is clear that the band profile is caused by a temperature-independent and carrierspecific broadening effect, presumably lifetime broadening. The observed bandwidth of $1.0 \mathrm{~nm}\left(\sim 35 \mathrm{~cm}^{-1}\right.$ around $\left.545 \mathrm{~nm}\right)$ corresponds to a lifetime of roughly $0.15 \mathrm{ps}$. The bandwidth profile does not allow conclusions on the nature of the laboratory carrier. The carrier must be a transient species (a molecular radical, a cation or anion, a weakly bound radical complex, possibly charged, or a vibrationally or electronically excited species) because no comparable spectra are recorded without plasma (i.e. with a regular $\mathrm{C}_{2} \mathrm{H}_{2} / \mathrm{He}$ expansion). The use of a $\mathrm{C}_{2} \mathrm{D}_{2} / \mathrm{He}$ expansion results in a red-shifted spectrum (Fig. 3), and from this it can be concluded that the laboratory carrier must contain both carbon and hydrogen. To check whether there are equivalent $\mathrm{H}$-atoms in this carrier, a $\mathrm{C}_{2} \mathrm{H}_{2} / \mathrm{C}_{2} \mathrm{D}_{2} 1: 1$ mixture in He has been used as an expansion gas, but this only results in a very broad absorption feature covering the whole region where results are found for a pure $\mathrm{C}_{2} \mathrm{H}_{2}$ and a pure $\mathrm{C}_{2} \mathrm{D}_{2}$ expansion. It is impossible to conclude anything about the actual number of equivalent $\mathrm{H}$-atoms in the carrier by determining the number of bands that show up, as could be demonstrated for $\mathrm{HC}_{6} \mathrm{H}^{+}$or $\mathrm{HC}_{7} \mathrm{H}$ (Sinclair et al. 1999; Ball et al. 2000a; Khoroshev et al. 2004). Also the use of another precursor (e.g. allene) did not provide conclusive information.

Additional experiments have been performed. The 543$545 \mathrm{~nm}$ region has been scanned using a two-photon REMPITOF experiment with the aim determining the mass of the carrier (Pino et al. 2001). No spectrum could be recorded, which may be related to the short lifetime of the excited state or with the fact that the carrier is an ion. Ions are indeed formed in this planar plasma source (Witkowicz et al. 2004). Both smaller and larger species have been observed, with optimum production rates depending, among other things, on the backing pressure. The production of larger species is generally more critical; e.g., higher backing pressures are needed, but this also may destabilize the plasma, which is unfortunate, particularly during long scan procedures. More complex species are generally found further downstream, but in this specific case, we did not observe large differences as a function of the distance from the laser beam to the nozzle orifice. This is the typical behaviour for a smaller constituent in the gas expansion. We tried to systematically study the voltage dependence of the signal. For a positive ion, an increase in voltage should go along with a decrease in signal for distances further downstream, as the jaws carry a negative voltage. It is the opposite for anions, but 10 years of experience with this source have shown that negative ions are rather hard to produce. Again, the changes we recorded were small and did not allow drawing hard conclusions. Following condition 2 mentioned in the introduction, we also searched in other wavelength regions blue-shifted by values typical for an excited $\mathrm{C}-\mathrm{C}, \mathrm{C}=\mathrm{C}$, $\mathrm{C} \equiv \mathrm{C}$, or $\mathrm{CH}$ stretch in the upper electronic state. Such excited bands have not been observed here, but it should be noted that these bands can be intrinsically weak.

In summary, we are left with a laboratory spectrum that coincides both in band maximum and band width with a known DIB band at $545 \mathrm{~nm}$. Our measurements show that the absorption spectrum of a transient molecule containing hydrogen and carbon reproduces the astronomical spectrum. The profile can be explained with life time broadening, and this is consistent with the observation that the laboratory and astronomical spectrum are identical; i.e., with no temperature constraints. In addition, it explains why the large bandwidth of this DIB does not vary along different lines of sight. The large effective absorption may also be indicative of an abundant carrier. The exact carrier, as such, remains an open question. The present result, however, may be useful for stimulating upcoming DIB work.

Acknowledgements. The results presented here bridge a period of 10 years. The cavity ring-down measurements were performed in the Institute for Physical Chemistry (Department of Chemistry, University of Basel) with support of the Swiss National Science Foundation, and the analysis follows recent observations and a collaboration within the framework of the FP6 research training network The Molecular Universe. Additional financial support of NOVA is gratefully acknowledged.

\section{References}

Araki, M., Linnartz, H., Kolek, P., et al. 2004, ApJ, 616, 1301

Ball, C. D., McCarthy, M. C., \& Thaddeus, P. 2000a, J. Chem. Phys., 112, 10149 Ball, C. D., McCarthy, M. C., \& Thaddeus, P. 2000b, ApJ, 529, L61

Bréchignac, P., \& Pino, T. 1999, A\&A, 343, L49

Cossart-Magos, C., \& Leach, S. 1990, A\&A, 233, 559

Foing, B. H., \& Ehrenfreund, P. 1994, Nature, 369, 296

Galazutdinov, G. A., Musaev, F. A., Krełowski, J., et al. 2000, PASP, 112, 648

Herbig, G. H. 1975, ApJ, 196, 129

Jenniskens, P., \& Desert, F. 1994, A\&AS, 106, 39

Jochnowitz, E. B., \& Maier, J. P. 2008, Mol. Phys., 106, 2093

Khoroshev, D., Araki, M., Kolek, P., et al. 2004, J. Molec. Spectrosc., 227, 81

Kirkwood, D. A., Linnartz, H., Grutter, M., et al. 1998, in Chemistry and Physics

of Molecules and Grains in Space, Faraday Discuss., 109, 109

Kroto, H. W., \& Jura, M. 1992, A\&A, 263, 275

Linnartz, H., Motylewski, T., \& Maier, J. P. 1998, J. Chem. Phys., 109, 3819

Linnartz, H., Motylewski, T., Vaizert, O., et al. 1999, J. Molec. Spectrosc., 197, 1

Maier, J. P., Lakin, N. M., Walker, G. A. H., et al. 2001, ApJ, 553, 267

McCall, B. J., Thorburn, J., Hobbs, L. M., Oka, T., \& York, D. G. 2001, ApJ, 559, L49

Motylewski, T., \& Linnartz, H. 1999, Rev. Sci. Instr., 70, 1305

Motylewski, T., Linnartz, H., Vaizert, O., et al. 2000, ApJ, 531, 312

Pino, T., Ding, H., Güthe, F., et al. 2001, J. Chem. Phys., 114, 2208

Raskin, G., \& Van Winckel, H. 2008, in SPIE Conf. Ser., 7014

Ruiterkamp, R., Halasinski, T., Salama, F., et al. 2002, A\&A, 390, 1153

Salama, F., Bakes, E. L. O., Allamandola, L. J., et al. 1996, ApJ, 458, 621

Salama, F., Galazutdinov, G. A., Krełowski, J., Allamandola, L. J., \& Musaev, F. A. 1999, ApJ, 526, 265

Sinclair, W. E., Pfluger, D., Linnartz, H., et al. 1999, J. Chem. Phys., 110, 296 Sorokin, P. P., Glownia, J. H., \& Ubachs, W. 1998, in Chemistry and Physics of Molecules and Grains in Space. Faraday Discuss., 109, 137

Thaddeus, P., \& McCarthy, M. C. 2001, Spectrochim. Acta, 57, 757

Tielens, A. G. G. M., \& Snow, T. P. 1995, The diffuse interstellar bands, ed.

A. G. G. M. Tielens, \& T. P. Snow, Astrophysics and Space Science Library, 202

Tuairisg, S. Ó., Cami, J., Foing, B. H., Sonnentrucker, P., \& Ehrenfreund, P. 2000, A\&AS, 142, 225

Tulej, M., Kirkwood, D. A., Pachkov, M., et al. 1998, ApJ, 506, L69

Witkowicz, E., Linnartz, H., de Lange, C., et al. 2004, Int. J. Mass Spectrom., 232,25 